\title{
Entretien avec Sylvie Tarozzi
}

Responsable du Pôle PronosTIC, FT R\&D

\section{(2) OpenEdition}

\section{Journals}

Édition électronique

URL : http://journals.openedition.org/communicationorganisation/3259

DOI : 10.4000/communicationorganisation.3259

ISSN : 1775-3546

Éditeur

Presses universitaires de Bordeaux

Édition imprimée

Date de publication : 1 juin 2005

ISSN : 1168-5549

\section{Référence électronique}

" Entretien avec Sylvie Tarozzi », Communication et organisation [En ligne], 27 | 2005, mis en ligne le 07

juin 2012, consulté le 03 mai 2019. URL : http://journals.openedition.org/

communicationorganisation/3259 ; DOI : 10.4000/communicationorganisation.3259

Ce document a été généré automatiquement le 3 mai 2019.

(C) Presses universitaires de Bordeaux 


\title{
Entretien avec Sylvie Tarozzi
}

\author{
Responsable du Pôle PronosTIC, FT R\&D
}

\section{NOTE DE L'ÉDITEUR}

Propos recueillis par Aurélie Laborde

1 Sylvie Tarozzi est ingénieur d'études en sociologie des usages et responsable du Pôle de recherche "PronosTIC: Outils et méthodes pour l'anticipation des services » à France Télécom R\&D.

2 Formée à l'Université de Grenoble (GRESEC), son premier contrat de recherche avec le CNET débute en 1993 et concerne l'insertion des mobiles dans les pratiques professionnelles de communication.

3 Sylvie Tarozzi poursuit ensuite sa carrière à France Télécom R\&D, d'abord comme « ergonome/sociologue » pour les services de télémédecine et santé, puis, depuis 1999, comme responsable d'unité de recherche.

Sylvie Tarozzi, pouvez-vous nous décrire en quelques mots l'organisation de France Télécom R\&D et le rôle de votre unité de recherche?

La Recherche est organisée en 16 Pôles. Chaque Pôle est animé par un expert et regroupe les chercheurs de la Division R\&D sur une large thématique scientifique. Il fournit un cadre de travail et d'échange entre les chercheurs.

Un programme de recherche est élaboré annuellement par chaque Pôle de Recherche sur des activités qui permettent à France Télécom de développer ou maintenir des compétences scientifiques de haut niveau sur des thématiques stratégiques en anticipation des besoins du Groupe.

Chaque Pôle dans son domaine doit identifier les évolutions et ruptures technologiques et en évaluer les conséquences sur les systèmes et services télécoms. A ces fins tout pôle se doit de tisser des partenariats scientifiques et techniques pour accélérer la mise en œuvre des innovations. 
Le Pôle de Recherche «PronoSTIC. Outils et méthodes pour l'anticipation des services " que je pilote, conduit des études autour de deux axes complémentaires: la recherche sur les outils et les méthodes de prospective, et la prospective amont et appliquée des services de télécommunications (exploration de nouveaux domaines émergents pour de nouveaux usages et construction d'une vision dynamique de l'offre à 5 et 10 ans). En amont des évolutions des pratiques de communication, les travaux de recherche s'attachent à détecter les évolutions et les tendances majeures et durables qui peuvent modifier ces pratiques (évolution sociales, technologiques, règlementaires, économiques,...). La prospective de services consiste à traduire les continuités ou les ruptures en nouveaux concepts de services en s'appuyant sur des approches pluridisciplinaires (ergonomie, sociologie, technologies, économie,...) et en mettant en œuvre des méthodes et des outils innovants pour en évaluer la pertinence et l'acceptabilité.

Pourquoi un pôle prospective?

Comment anticiper dans un environnement de plus en plus complexe où les risques de discontinuités et de ruptures dans tous les domaines se multiplient?

L'accélération de la complexité du monde qui nous entoure favorise l'incertitude voire l'insécurité, et la vitesse à laquelle se produisent les changements se traduit pour le «R\&D» par le raccourcissement des cycles de conception et par la recherche d'une meilleure adéquation entre l'offre et la demande.

A ce titre les questions d'appropriation d'un dispositif dans la vie quotidienne d'un utilisateur restent centrales. Mais en amont, il existe une période, un lieu, un temps de négociation entre l'utilisateur, ses pratiques, ses comportements et les représentations et imaginaires qu'il se fait du produit et du service (et même du concept) et de son usage potentiel. La question de l'acceptabilité devient dès lors capitale, dans cette phase qui précède l'usage. Cette approche par le «faire sens » prend toute sa valeur dans la dynamique de l'innovation poussée par les technologies et qui intègre difficilement les usages. Il est essentiel, d'une part, d'évaluer le champ d'acceptation sociale, c'est à dire les limites, les seuils et les conditions, soit l'expérience de l'utilisateur potentiel et d'autre part de co-produire avec lui les usages de cette technologie.

Cette démarche suppose des méthodes de mise en perspective des innovations technologiques et des évolutions sociales (styles de vie, valeurs, opinions, modes de consommation) aptes à interpréter et intégrer les différentes dynamiques de changements voire de ruptures sociales ou technologiques.

Dans cette perspective, les travaux de recherche du Pôle sont pluridisciplinaires, mettant en œuvre par nature des disciplines scientifiques multiples : la sociologie des usages, la sociologie de l'innovation, l'économie, l'ergonomie de conception, le marketing amont,... associées aux évolutions technologiques.

Quels sont aujourd'hui les programmes en cours dans votre unité de recherche?

Le premier axe du programme concerne l'acquisition de compétences en matière d'outils et de méthodes de prospective orientée services.

Le deuxième axe, «Prospective des services », comprend des recherches de prospective sur les thématiques connues et développées dans les laboratoires de R\&D mais aussi des 
études prospectives plus exploratoires portant sur des thématiques émergentes à 5 ou 15 ans (le Développement Durable, les applications des nano et biotechnologies).

Pouvez vous nous donner quelques exemples de travaux de recherche?

- Citoyenneté numérique, e-démocratie, e-gouvernement en France (TIC au niveau local et national) et à l'international.

- Expérimentations et évaluations sociologiques et ergonomiques des systèmes de vote électronique.

- TIC et lien social : associations de patients et d'apprenants, militantisme international, TIC et sport

- TIC et développement durable : dimensions développement des territoires, énergies, impacts sociaux des TIC et développement social

- TIC et applications des nano et biotechnologies

- Visions des services à horizon 5 à 10 ans. Nouveaux concepts de services : éducation, santé, l'éducation et la formation, transport, habitat, entreprise...

- Travaux sur les capteurs embarqués sur la personne ou disséminés dans les environnements physiques.

- Travaux sur la transmission des cinq sens (multisensorialité)

- Recherches spécifiques sur les outils et les méthodes de prospective appliquée

- Des thématiques transverses comme l'acceptabilité sociale des TIC.

Ces programmes de recherche sont-ils réalisés par FT R\&D seul ou développés en partenariat?

Les partenariats et les collaborations se font majoritairement avec les laboratoires universitaires ou publics (laboratoire "Territoire», Université Joseph Fourier; CEDEPIC, Université Grenoble 3; IRUTIC, Université Rennes 2; CERAG, Université Grenoble 2; Ecole polytechnique de Lausanne, INT Paris ; PACTE, IEP Grenoble ; ESC Sophia Antipolis; UNSA, Sophia Antipolis; LI2G et TIMC, UJF; ENST Bretagne; laboratoire LUCE du CNRS; MSH Rhône-Alpes; Higher School of Management, Varsovie). Ils s'élargissent en 2005 à des partenariats industriels.

Le thème du dossier de ce numéro de la Revue concerne les "nouvelles » formes de relations consommateurs/entreprises. Votre démarche, prenant en compte le consommateur, client ou futur client bien en amont de la création de services, semble parfaitement illustrer cette réflexion. Quel est votre point de vue sur cette question?

A France Télécom, nous observons l'usager/client à l'aide de nombreux dispositifs : outils de CRM, enquêtes clientèles, analyse des pratiques et traces de communication (flux de communication), enquêtes qualitatives,

La R\&D tend à intégrer toujours plus les clients potentiels dans ses propres processus de conception des services à travers un ensemble varié de méthodologies (le pluralisme méthodologique est alors indispensable) qui font appel à la participation des utilisateurs : analyse de l'activité et des usages, méthodes de créativité et de validation par les clients, tests ergonomiques, suivis d'expérimentations, etc.).

De nombreuses études conduites en 2001-2003 ont fait apparaître les transformations de la figure de l'usager traditionnel en utilisateur et en client dans la "société de l'information ». Des enquêtes auprès d'associations militantes, internationales ou 
sectorielles (santé, éducation, sport) ont montré que l'utilisateur ne pouvait être simplement réduit à un client cible en bout de chaîne du processus de conception. Par exemple, les secteurs de la santé ou de la formation sont marqués par des stratégies de coopération et de regroupement dans des réseaux d'intérêt commun en vue d'augmenter les capacités des utilisateurs à se faire entendre, à centraliser les moyens et à professionnaliser leurs actions. Ces collectifs d'utilisateurs se perçoivent souvent eux-mêmes comme des acteurs à part entière du processus d'innovation, développant une forte capacité de réflexion et de critique face aux experts et aux professionnels. A l'évidence, ces pratiques collectives, leur inventivité, ces manières de gérer les contraintes et d'associer les services constituent autant de sources d'innovation incontournables.

Dans la société civile, les capacités d'inventivité des utilisateurs sont à l'origine de biens des innovations très récentes.

La mise en évidence des capacités d'innovation des utilisateurs (inventivité et créativité) ainsi que du processus émergent de décloisonnement interne et externe entre offre et demande (modification du rapport expert/profane) dans la production de nouveaux services ouvre la voie à une refondation de nos méthodes et outils de conception. Il apparaît donc nécessaire de développer une connaissance amont des innovations émergentes qu'elles soient sociales ou techniques et d'interroger les processus d'innovation dans différents groupes sociaux.

Comment voyez-vous évoluer cette relation client/entreprises?

La question du «client » est la meilleure expression de l'hypothèse d'une tendance croissante à l'intégration des entreprises et des marchés. Nous allons assister à une accélération du processus d'intégration avec d'une part l'entrée du client dans l'organisation des entreprises, son ascension dans l'ensemble de ses fonctions puis à son arrivée dans une co-conception et d'autre part une démarche de personnalisation du client par le marketing.

Quel est ce client que l'entreprise projette de satisfaire et de personnaliser jusqu'à le faire participer à la conception des produits et des services?

Dans les entreprises, la co-conception au sens strict est une toute nouvelle démarche. Elle est loin d'être aujourd'hui une pratique courante alors que la remontée du client vers la conception est le résultat d'une longue histoire.

La co-conception participative (co-design ou concurrent engineering), qui consiste à intégrer tous les participants à la conception jusqu'aux utilisateurs potentiels est une réponse que les entreprises vont tester dans leurs processus d'innovation.

Cela pourra constituer un des éléments essentiels à la tendance d'intégration des entreprises et des marchés. Les méthodologies de prospective et d'innovation de services devraient en être transformées.

La prospective de services à France Télécom R\&D se dote progressivement d'outils et de méthodes d'analyse des modes de production de l'innovation sociale, afin de réadapter et de préciser de façon continue la connaissance et l'intégration du client dans la conception de services. 
Quelles sont les recherches que vous menez actuellement sur cette thématique, pourriez vous nous donner quelques exemples?

Nos recherches dans ce domaine s'organisent autour de trois grandes études.

Innov'acteurs, études des pratiques d'innovation sociale dans les groupes sociaux marginaux :

L'étude Innov'acteurs porte sur l'exploration des modes de production d'innovations sociales des groupes marginaux pour comprendre et anticiper les méthodes de conception qui préconisent la participation des futurs utilisateurs

Dans cette perspective, une importante enquête de terrain a débuté en juillet 2004, auprès de sept groupes sociaux en apparence hétéroclites retenus pour leurs capacités présumées à renouveler et créer des formes de participation propres aux finalités qui les réunissent.

Sur la base d'entretiens semi-directifs, de méthodes d'observation participante et de la confrontation de monographies descriptives, nous avons commencé par identifier les caractéristiques des modes de production d'innovations sociales de ces différents groupes (qu'ils soient formels ou informels). Pour cela, nous nous appuyons sur le concept de norme sociale qui permet de formaliser les processus d'exclusion et d'inclusion à l'œuvre dans ces différents groupes sociaux.

Nous faisons l'hypothèse que la question de l'intégration dans des systèmes de normes sociales (normes de consommation, normes d'utilisation, normes institutionnelles, normes familiales, etc.) permet précisément à ces groupes d'individus, généralement marginaux, de penser implicitement ou explicitement la production de solutions innovantes : organisation du groupe, participation au renouvellement et à la définition des règles de fonctionnement du groupe, production d'outils.

Ces travaux se poursuivent en 2005 et nous escomptons ainsi enrichir nos études en prospective de service et en co-conception en intégrant des critères sur les motivations, mécanismes et économies des pratiques collectives des TIC.

De la co-conception à l'intégration du client dans le système entreprise/marché :

(Partenariat avec le CEDEPIC, étude piloté par Bernard Floris, contrat de recherche et thèse associée (Alexandra Peronnet))

L'économie globalisée se caractérise par une interaction croissante entre entreprise et marché, production et consommation. L'entreprise étendue désigne ainsi ce processus de décloisonnement interne et externe. C'est dans le secteur des activités de services, et en particulier dans celui de l'information et de la (télé) communication que cette interaction est la plus développée et la plus stratégique en terme de création de valeur. Si les entreprises s'adaptent toujours mieux aux attentes des clients, elles recherchent parallèlement une adaptation des clients à leur processus de mise en marché. La logique client conduit à structurer l'organisation flexible en fonction des variations de la consommation, mais elle consiste aussi à produire l'univers pratique et symbolique suscitant le besoin des produits proposés par les entreprises. La prospective d'innovation en R\&D, le marketing (en particulier direct, expérientiel et branding), et le CRM sont les outils les plus performants de ce processus.

Cette tendance ne peut être comprise uniquement par l'analyse des usages dans différents segments existants ou à construire. La compréhension de l'univers mental 
(émotionnel et culturel) des utilisateurs, bien au-delà de l'usage lui-même (valeurs, consommation, hédonisme, individualisme, autonomie, etc.) doit être croisée avec celle des usages.

La recherche se propose donc de saisir d'une part les processus d'intégration de l'entreprise étendue à son marché et à ses clients (transformations induites parallèlement dans l'organisation du travail de production et du travail de consommation), et d'autre part, le croisement des usages et des représentations des clients dans le cadre de la création de nouveaux produits.

CLICOS: Méthodes de prospective et pratiques de représentations du client coconcepteur de services

En complémentarité avec les travaux précédents, il s'agit de comprendre ici comment les entreprises mettent en place des processus et des méthodes qui leur permettent de décliner une stratégie de co-conception et de connaissance client. Quels objectifs? Quelles méthodes? Quels outils? Au service de quelle stratégie?

Cette recherche porte sur un état des lieux des méthodes de co-conception à FT R\&D, sur une analyse des représentations du client dans la fonction Marketing et des pratiques et méthodologies de représentation du client co-concepteur de service.

Pour conclure, je souhaiterais revenir sur les études d'acceptabilité autour des TIC, ont-elles véritablement un intérêt aujourd'hui et influencent elles les industriels et opérateurs?

La question de l'acceptabilité prend toute son importance si l'on considère le contexte social très sensible aux retombées de chaque avancée technique et des rapports redéfinis entre la technique et la société. Il y a d'une part du coté des citoyens la montée en exigence en matière de protection et de sécurité civile, la demande de participation aux choix technologies, l'application du principe de précaution et d'autres part des techniques de plus en plus invasives, touchant la personne et son intégrité.

Quand on interroge les représentations des usages des nouvelles technologies, du point de vue de l'utilisateur nous entrons dans un univers d'attentes ambivalentes, de paradoxes qui peuvent être autant de limites à l'acceptabilité sociale des TIC car pour être efficaces celles-ci sont de plus en plus invasives.

Finalement, les TIC apparaissent dans le cadre de la mise en réseau généralisée qu'elles supposent sous des interprétations très ambivalentes voire paradoxales: on peut leur attribuer un rôle dans la redéfinition des échanges plus simples, plus rapides, plus ouverts, plus sûrs mais elles peuvent aussi être contestées dans leurs fonctions d'intrusion, y compris de malveillante (hacking, escroquerie), de capture et de surveillance des existences.

Dans cette perspective, nous avons engagé des travaux de recherche sur des technologies telles que la localisation, l'image et les identités, la traçabilité, l'anonymat, etc... il s'agit pour nous d'identifier les représentations propres aux TIC en fonction de trois formes repérées : les représentations générales, les attentes de la technique et les représentations des pratiques. 